\title{
Anomalous weak values are caused by disturbance
}

\author{
Asger C. Ipsen
}

January 21, 2022

\begin{abstract}
In combination with post-selection, weak measurements can lead to surprising results known as anomalous weak values. These lie outside the bounds of the spectrum of the relevant observable, as in the canonical example of measuring the spin of an electron (along some axis) to be 100. We argue that the disturbance caused by the weak measurement, while small, is sufficient to significantly affect the measurement result, and that this is the most reasonable explanation of anomalous weak values.
\end{abstract}

\section{Introduction}

The subject we want to address can be somewhat confusing at the conceptual level. So, before getting to the actual arguments, we will start with a rather long introduction where we try to explain the main issues as clearly as possible.

For concreteness we will discuss weak values in the context they where originally introduced 1,3 , namely that of a weakly interacting limit of a von Neumann measurement. Let us begin by recalling the standard von Neumann model. It describes the measurement of an observable $\hat{A}$ as a two-step process; first the system of interest, initially in state $|\psi\rangle$, interacts with the measurement apparatus (or meter) according to

$$
|\psi\rangle|I\rangle \rightarrow e^{-i \lambda \hat{A} \hat{p}}|\psi\rangle|I\rangle
$$

The meter is modeled by a single canonical degree of freedom $[\hat{x}, \hat{p}]=i$ and, for the purposes of this paper, we will take the initial state $|I\rangle$ to be a Gaussian with zero mean and unit variance,

$$
\langle x \mid I\rangle:=\sqrt{G(x)}, \quad G(x):=(2 \pi)^{-1 / 2} e^{-x^{2} / 2} .
$$

Asger C. Ipsen

Farum, Denmark E-mail: acipsen@gmail.com 
The parameter $\lambda \in \mathbb{R}$ controls the strength of the interaction. Note that we will always assume that the Hamiltonian of the isolated system vanishes. The procedure is completed by a sharp measurement of $\hat{x}$, resulting in $x$ with probability (we assume that the system is finite dimensional)

$$
\begin{aligned}
P_{\lambda}(x \mid \psi) & =\sum_{i}\left|\left\langle a_{i}\left|\left\langle x\left|e^{-i \lambda \hat{A} \hat{p}}\right| \psi\right\rangle\right| I\right\rangle\right|^{2} \\
& =\sum_{i}\left|\left\langle a_{i} \mid \psi\right\rangle\right|^{2}\left|\left\langle x\left|e^{-i \lambda a_{i} \hat{p}}\right| I\right\rangle\right|^{2} \\
& =\sum_{i}\left|\left\langle a_{i} \mid \psi\right\rangle\right|^{2} G\left(x-\lambda a_{i}\right)
\end{aligned}
$$

For sufficiently large $\lambda$ the different peaks of the distribution can be resolved, and a result $x \simeq \lambda a_{i}$ corresponds to a specific eigenvalue of $\hat{A}$.

In the opposite limit of small $\lambda$ the peaks corresponding to different eigenvalues combine to a single peak centered on the expectation value of $\hat{A}$,

$$
P_{\lambda}(x \mid \psi)=G\left(x-\lambda\langle\hat{A}\rangle_{\psi}\right)+O\left(\lambda^{2}\right), \quad\langle\hat{A}\rangle_{\psi}:=\langle\psi|\hat{A}| \psi\rangle .
$$

We will use the term weak measurement to mean that $\lambda$ is sufficiently small that $O\left(\lambda^{2}\right)$ terms can be neglected. To obtain the weak value we extend the measurement procedure just described by performing a post-selection on the system after the von Neumann interaction (11). That is, we perform a projective measurement with $|\varphi\rangle$ as a one of the eigenstates, and only keep the measurement outcomes for the runs of the experiment where the result of the final measurement is $|\varphi\rangle$. The resulting distribution of $x$ conditioned on successful post-selection is

$$
\begin{aligned}
P_{\lambda}(x \mid \varphi, \psi) & =P_{\lambda}(\varphi \mid \psi)^{-1}\left|\left\langle\varphi\left|\left\langle x\left|e^{-i \lambda \hat{A} \hat{p}}\right| \psi\right\rangle\right| I\right\rangle\right|^{2} \\
& =G\left(x-\lambda \operatorname{Re} \hat{A}_{w}\right)+O\left(\lambda^{2}\right)
\end{aligned}
$$

with the weak value $\hat{A}_{w}$ defined by

$$
\hat{A}_{w}:=\frac{\langle\varphi|\hat{A}| \psi\rangle}{\langle\varphi \mid \psi\rangle} .
$$

The normalization factor is the unconditional post-selection probability $P_{\lambda}(\varphi \mid \psi)=$ $|\langle\varphi \mid \psi\rangle|^{2}+O(\lambda)$. We have assumed that $|\varphi\rangle$ is not orthogonal to $|\psi\rangle$, and we will continue to do so for the remainder of the paper.

The purpose of the interaction (11) is to displace the meter observable $\hat{x}$ proportionally to the system observable $\hat{A}$, but it will in general also cause some uncontrolled change in the state of the system itself. This is the backaction or disturbance of the measurement. The parameter $\lambda$ directly controls the strength of the interaction, and so, for weak measurements where $\lambda$ is small, the amount of disturbance is also small. The central question we are interested in is whether it is small enough to be neglected. More precisely, we 
want to investigate the hypothesis of

Disturbance Insignificance (DI): To linear order in $\lambda$ the disturbance (of the system) caused by the intermediate weak measurement can be neglected. In particular, the order $\lambda$ shift of the conditional $x$ distribution (5) is not affected by the disturbance.

An alternative formulation of DI is given in Appendix A, and a more general hypothesis is discussed in Appendix D.

A Fourier transformation of (2) shows that the distribution of $p$ is Gaussian with zero mean and a variance of $1 / 4$. Substituting a normal distributed random number $p$ for $\hat{p}$ as a heuristic model, we would then expect the back-action of (1), in a given run of the experiment, and for small $\lambda$, to be roughly

$$
\Delta|\psi\rangle \sim-i \lambda p \hat{A}|\psi\rangle .
$$

This is of the same order of magnitude as the shift of the meter (i.e. $\lambda$ ), so it is not a priori clear that DI is a good assumption. The main claim of the present paper is that DI does not follow from ordinary quantum mechanics, but that it rather is an additional postulate which leads to surprising or paradoxical results in some situations.

A central concept in the weak value literature is that of a pre- and postselected (PPS) ensemble. Given a number of identical systems all prepared in the state $|\psi\rangle$ at time $t_{1}$, consider the subensemble of systems found to be in state $|\varphi\rangle$ when we make a projective measurement on each system at a later time $t_{2}$. This subensemble is said to be the PPS ensemble defined by states $|\psi\rangle$ and $|\varphi\rangle$. Note that no other interaction with the systems is to take place between $t_{1}$ and $t_{2}$. This ensemble is unusual in that, as soon as we learn which systems belong to it, i.e. at $t_{2}$, the systems are strongly and irreversibly perturbed by the projective measurement. We cannot make an ordinary measurement at an intermediate time $t, t_{1}<t<t_{2}$, since doing so would influence the post-selection. However, if we assume DI, we should be able to perform a weak measurement of $\hat{A}$ on each system without significantly altering the ensemble. We are then naturally lead to consider the average of the measurement results corresponding to post-selected systems as a kind of expectation value of $\hat{A}$ in the PPS ensemble 1 This average is just the conditional expectation value which, according to (5), is (proportional to) the real part of $\hat{A}_{w}$.

It is important to note that the understanding of the weak value as the result of a measurement of $\hat{A}$ in a PPS ensemble necessarily rests on DI. Without DI the weak measurement must be considered an active part of the process that selects the members of the ensemble. But then it is not reasonable to think of the PPS ensemble as an independently existing thing on which we perform some measurement 2 For this reason we claim that, while a weak

\footnotetext{
1 For example, it is stated that "[...] we can operationally interpret the real part of the weak value [...] as an idealized conditioned average of $\hat{A}$ in the limit of zero measurement disturbance." in Ref. [11.

2 In the case of strong intermediate measurements, a similar objection regarding postselected ensembles was raised in Ref. [5].
} 
measurement by itself constitute a legitimate, if imprecise, measurement, the combination of a weak measurement and post-selection should not be considered as any kind of measurement of $\hat{A} 3$

The issue might by illustrated by a simple example[1. Suppose we, instead of post-selecting on the system, decide to only keep data from runs with, say, $x \geq 100 \lambda$. Then the conditional distribution becomes

$$
P(x \mid " x \geq 100 \lambda ", \psi)=\left\{\begin{array}{ll}
\mathcal{N} G\left(x-\lambda\langle\hat{A}\rangle_{\psi}\right)+O\left(\lambda^{2}\right), & x \geq 100 \lambda \\
0, & x<100 \lambda
\end{array},\right.
$$

with $\mathcal{N}$ a normalization constant. Clearly the expectation value of $x$ would be larger than $100 \lambda$ in this case, but no one would interpret this to mean that we have constructed an ensemble where the expectation value of $\hat{A}$ is larger than 100. It would also not be reasonable to claim that this procedure constitutes a measurement of $\hat{A}$ in any sense, since we are clearly changing the result by "cherry picking" data points. This example is so different from the actual protocol for obtaining the weak value that the comparison might seem silly. However, note that the interaction (11) perturbs the system in a way which depends on the state of the measurement apparatus. This means that, by post-selecting on the system, one is also indirectly post-selecting on the meter. So post-selecting on a observable of the system might have some similarities with our artificial example of post-selecting on the position of the meter after all.

Returning to weak values, the crucial point is that, since the post-selection occurs after the weak measurement, the interaction between system and measurement apparatus can directly influence the probability of successful postselection. This can lead to unintuitive statistical effects. When $\lambda$ is made very small, so as to disturb the system very little, the amount of information contained in the data of each experimental run also becomes very small. Our basic conclusion can then be phrased as: The statistical amplification (by combining data from many runs of the experiment) necessary to determine the weak value is also sufficient to amplify these small unintuitive effects caused by the disturbance of the intermediate weak measurement. A number published works contain statements that appear to contradict our findings. We will not attempt an exhaustive review of the literature, but a representative list of examples is Refs. [23, 20, 22, 14, 11, 4, 24, 7], see also Appendix [B] It should be noted that a few authors have, by different arguments, reached conclusions similar to ours $15,13,17$.

A final notion we need to introduce is that of an anomalous weak value. The ordinary expectation value $\langle\hat{A}\rangle$ is bounded between the smallest and largest eigenvalue of $\hat{A}$. On the other hand, it is always possible 4 to find states $|\psi\rangle$,

\footnotetext{
3 A post-selected weak measurement is certainly a legitimate experimental procedure which leads to the conditional expectation value $\operatorname{Re} \hat{A}_{w}$. However, without the assumption of DI, it is not reasonable to interpret this value as the result of a measurement of $\hat{A}$.

4 As long as $\hat{A}$ is not proportional to the identity, one can find states $|\psi\rangle,|\perp\rangle$ such that $\langle\perp \mid \psi\rangle=0$ and $\langle\perp|\hat{A}| \psi\rangle>0$. With $|\varphi\rangle=\sqrt{1-\epsilon^{2}}|\perp\rangle+\epsilon|\psi\rangle$ and $\epsilon \in[-1,1] \backslash\{0\}$ sufficiently small, $\operatorname{Re} \hat{A}_{w}=\epsilon^{-1}\langle\perp|\hat{A}| \psi\rangle+O\left(\epsilon^{0}\right)$ is anomalous.
} 
$|\varphi\rangle$ such that $\operatorname{Re} \hat{A}_{w}$ is outside these bounds. When this happens we say that the weak value is anomalous. This is a striking and unintuitive phenomenon which was even the basis for the title of the first paper on weak values [1].

From the point of view that the weak value is a kind of expectation value it is difficult to accommodate such anomalous values. Indeed, suppose we have $N$ copies of the system prepared in the state $|\psi\rangle$ and we perform an ordinary (strong) measurement of $\hat{A}$ on each. Each measurement results in some eigenvalue $a_{n}, n=1, \ldots, N$. One could now argue that there should be some subensemble $\mathcal{S} \subseteq\{1, \ldots, N\}$ (of size $|\mathcal{S}| \sim N|\langle\varphi \mid \psi\rangle|^{2}$ ) consisting of those systems which would have been post-selected in state $|\varphi\rangle$, had we not performed the intermediate measurement. In other words, the members of $\mathcal{S}$ constitute a realization of the PPS ensemble. The expectation value of $\hat{A}$ in $\mathcal{S}$ could then be given its usual meaning as the empirical mean

$$
\lim _{N \rightarrow \infty} \frac{1}{|\mathcal{S}|} \sum_{n \in \mathcal{S}} a_{n},
$$

but this quantity is clearly bounded like the ordinary expectation value, and thus cannot in general be identified with $\operatorname{Re} \hat{A}_{w}$. Note that it is not possible to compute the average (9) in an actual experiment, since measuring $\hat{A}$ (strongly) makes it impossible to determine $\mathcal{S}$. Now, if one, as we are advocating, understands the shift in the distribution of $x$ as partly due to post-selection effects, the phenomenon of anomalous weak values becomes a non-mystery. Indeed, there appears to be no reason that such statistical effects should be bounded by the spectrum of $\hat{A}$. In the next section we will see in an explicit example that disturbance and post-selection can in fact lead to this kind of unbounded shift.

The remainder of the paper is structured as follows: Section 2 contains the main argument. We first demonstrate that, in a certain sense, the mathematical structure of quantum mechanics allows the disturbance caused by a weak interaction to appear smaller than it really is. Then we show that the amount of disturbance caused by a weak measurement is sufficient, in combination with post-selection, to cause shifts in the conditional $x$ distribution of magnitude $\lambda$ (i.e. of the same order as the shift in Eq. (5)). From considerations of sequential weak measurements, an independent argument against DI is given in Section 3. Appendix A contains some remarks on the notion of measurement disturbance and a reformulation of DI, while Appendix B quotes some passages from the literature that seem to endorse the hypothesis of DI. Appendix C extends the reasoning of Section 2 to the case where an entire ensemble is measured by a single measurement apparatus, and Appendix D discusses some related more technical work due to Dressel and Jordan.

\section{The significance of a little disturbance}

We begin by considering the unconditional probability $P_{\lambda}(\varphi \mid \psi)$ of successful post-selection more carefully. It is clear that any deviation from its un- 
perturbed value $|\langle\varphi \mid \psi\rangle|^{2}$ must be due to back-action from the intermediate measurement. Expanding in the coupling $\lambda$ we find

$$
\begin{aligned}
P_{\lambda}(\varphi \mid \psi) & :=\int \mathrm{d} x\left|\left\langle\varphi\left|\left\langle x\left|e^{-i \lambda \hat{A} \hat{p}}\right| \psi\right\rangle\right| I\right\rangle\right|^{2} \\
& =\int \mathrm{d} x G(x)\left|\left\langle\varphi\left|\left(1+\lambda \hat{A} \frac{x}{2}+\frac{\lambda^{2}}{2} \hat{A}^{2}\left[\frac{x^{2}}{4}-\frac{1}{2}\right]+O\left(\lambda^{3}\right)\right)\right| \psi\right\rangle\right|^{2} \\
& =|\langle\varphi \mid \psi\rangle|^{2}\left[1+\frac{\lambda^{2}}{4}\left(\left|\hat{A}_{w}\right|^{2}-\operatorname{Re}\left[\left(\hat{A}^{2}\right)_{w}\right]\right)+O\left(\lambda^{4}\right)\right],
\end{aligned}
$$

where

$$
\left(\hat{A}^{2}\right)_{w}:=\frac{\left\langle\varphi\left|\hat{A}^{2}\right| \psi\right\rangle}{\langle\varphi \mid \psi\rangle} .
$$

The leading correction is of order $\lambda^{2}$, which suggests that back-action is a second order effect. It thus appears as if the disturbance really is insignificant in the $\lambda \rightarrow 0$ limit 5 Despite the intuitive appeal of this reasoning, it is in fact misleading, as we will now argue.

Consider what would happen if we, instead of the weak measurement, simply gave the system a small unitary kick

$$
|\psi\rangle \rightarrow e^{-i \lambda \hat{A} x^{\prime} / 2}|\psi\rangle
$$

with $x^{\prime}$ drawn at random from the standard Gaussian distribution $G\left(x^{\prime}\right)$. The post-selection probability is seen to be

$$
\begin{aligned}
P_{\lambda}^{\prime}(\varphi \mid \psi) & :=\int \mathrm{d} x^{\prime} G\left(x^{\prime}\right)\left|\left\langle\varphi\left|e^{-i \lambda \hat{A} x^{\prime} / 2}\right| \psi\right\rangle\right|^{2} \\
& =|\langle\varphi \mid \psi\rangle|^{2}\left[1+\frac{\lambda^{2}}{4}\left(\left|\hat{A}_{w}\right|^{2}-\operatorname{Re}\left[\left(\hat{A}^{2}\right)_{w}\right]\right)+O\left(\lambda^{4}\right)\right]
\end{aligned}
$$

exactly as in (10). We see that the disturbance is able to "hide"; in each run of the experiment the system received a kick of magnitude $\lambda$, but, after marginalizing over $x^{\prime}$ to get the unconditional probability (13), the linear term drops out.

We can take this example further by considering the conditional distribution of $x^{\prime}$,

$$
\begin{aligned}
P^{\prime}\left(x^{\prime} \mid \varphi, \psi\right) & :=P_{\lambda}^{\prime}(\varphi \mid \psi)^{-1} G\left(x^{\prime}\right)\left|\left\langle\varphi\left|e^{-i \lambda \hat{A} x^{\prime} / 2}\right| \psi\right\rangle\right|^{2} \\
& =G\left(x^{\prime}-\lambda \operatorname{Im} \hat{A}_{w}\right)+O\left(\lambda^{2}\right) .
\end{aligned}
$$

Notice the similarity to (5), even though no measurement of $\hat{A}$ is taking place. The distribution (14) shows very explicitly that the disturbance (12) is significant, since it is entirely responsible for the shift. This also means that we cannot conclude from the smallness of the correction found in Eq. (10) that

${ }^{5}$ Variants of this argument appears several places, e.g. [22,14,4,7. 
DI holds. The shift $\operatorname{Im} \hat{A}_{w}$, like $\operatorname{Re} \hat{A}_{w}$, can be made arbitrarily large by appropriate choice of $|\psi\rangle$ and $|\varphi\rangle$. As the shift is clearly a statistical effect in this case, it is not surprising that it can exceed the spectrum of $\hat{A}$.

To make further contact between our 'fake measurement' and an actual weak measurement, it is useful to define the operator

$$
\hat{x}^{\prime}:=2 \hat{p}
$$

Our choice of $|I\rangle$ is such that the wavefunction in $\hat{x}^{\prime}$-basis is identical to that in $\hat{x}$-basis,

$$
\left\langle x^{\prime} \mid I\right\rangle=\sqrt{G\left(x^{\prime}\right)} .
$$

We then see that $P_{\lambda}^{\prime}(\varphi \mid \psi)$ can be expressed as

$$
P_{\lambda}^{\prime}(\varphi \mid \psi)=\int \mathrm{d} x^{\prime}\left|\left\langle\varphi\left|\left\langle x^{\prime}\left|e^{-i \lambda \hat{A} \hat{p}}\right| \psi\right\rangle\right| I\right\rangle\right|^{2}
$$

It is now clear that $P_{\lambda}^{\prime}(\varphi \mid \psi)=P_{\lambda}(\varphi \mid \psi)$ for any $\lambda$, since the only difference between (10) and (17) is whether we trace out the meter in $\hat{x}$-basis or $\hat{x}^{\prime}$ basis. Similarly we find that $P^{\prime}\left(x^{\prime} \mid \varphi, \psi\right)$ is equal to the conditional probability distribution for a measurement of $\hat{x}^{\prime}$,

$$
P_{\lambda}^{\prime}\left(x^{\prime} \mid \varphi, \psi\right)=P_{\lambda}(\varphi \mid \psi)^{-1}\left|\left\langle\varphi\left|\left\langle x^{\prime}\left|e^{-i \lambda \hat{A} \hat{p}}\right| \psi\right\rangle\right| I\right\rangle\right|^{2}
$$

The fact that the shift of $\hat{p} \propto \hat{x}^{\prime}$ is proportional to $\operatorname{Im} \hat{A}_{w}$ was already mentioned in Footnote 4 of [1].

We now have two formulations of our fake weak measurement, both leading to the same probabilities. One is in terms of a random unitary kick (12), the other keeps the von Neumann interaction (1), but we measure the meter observable $\hat{x}^{\prime}$ instead of $\hat{x}$. The reason that these lead to the same probabilities, is that $\left|x^{\prime}\right\rangle$ is an eigenstate of $\exp (-i \lambda \hat{A} \hat{p})\left(\right.$ since $\left.\hat{x}^{\prime} \propto \hat{p}\right)$, and that one can thus interchange the order of the interaction with the system and the measurement of $\hat{x}^{\prime}$.

Let us make a couple of further remarks:

1) The fact that the distribution of a variable is shifted in some process involving post-selection does not mean that the underlying physical quantity was changed by the same amount (on average). In our example the value of $x^{\prime}$ is chosen before the interaction with the system, and it is never changed from this original value. Nevertheless, the conditional distribution is shifted according to Eq. (14). Returning to weak measurements, it follows that we cannot conclude from (5) that the position of the meter is shifted by $\lambda \operatorname{Re} \hat{A}_{w}$ (on average). We thus disagree with the claim of Ref. 22 that the "weak value can be regarded as a definite mechanical effect on a measuring probe...".

2) An interaction with the system which changes the post-selection probability to the one given in Eq. (10) is able to shift the distribution of $x$ an

\footnotetext{
6 This follows by the argument of Footnote 4 but with $|\varphi\rangle=i \sqrt{1-\epsilon^{2}}|\perp\rangle+\epsilon|\psi\rangle$.
} 
amount of order $\lambda$ purely through post-selection effects. Indeed, take the interaction

$$
|\psi\rangle|I\rangle \rightarrow e^{-i \lambda \hat{A} \hat{x} / 2}|\psi\rangle|I\rangle
$$

By the above consideration, with $\hat{x}$ and $\hat{x}^{\prime}$ interchanged, this leads to the postselection probability (10) and a conditional $x$ distribution which is shifted by $\lambda \operatorname{Im} \hat{A}_{w}$.

3) An interesting situation arises when the choice between measuring $\hat{x}$ and $\hat{x}^{\prime}$ is delayed to after post-selection of the system. If the choice falls on $\hat{x}^{\prime}$ we have seen that the disturbance must be considered significant. On the other hand, according to DI, when measuring $\hat{x}$ we should be able to neglect the disturbance. But this would mean that, on assumption of DI, the experimenter is able to choose, after the post-selection has taken place, whether said postselection was significantly disturbed. It is not at all clear how one is to make sense of this conclusion.

In summary we have failed to find any evidence for DI, i.e. that the disturbance caused by the von Neumann interaction (1) can be neglected. Instead it appears natural to assume that this disturbance plays a significant role in the observed shift in the conditional distribution of the meter observable $\hat{x}$. This is also sufficient to explain why the weak value can exceed the spectrum of $\hat{A}$ under certain circumstances.

\section{Sequential weak measurements}

An interesting situation where the presence of significant back-action becomes apparent, is the case of sequential weak measurements. That is, when several independent weak measurements are performed on the system between preparation and post-selection 21,19]. For our purposes it is sufficient to consider two measurements. The setup then consist of two identically prepared meters, and the combined interaction corresponding to first measuring $\hat{A}$ then $\hat{B}$ is

$$
|\psi\rangle|I\rangle_{1}|I\rangle_{2} \rightarrow e^{-i \lambda_{2} \hat{B} \hat{p}_{2}} e^{-i \lambda_{1} \hat{A} \hat{p}_{1}}|\psi\rangle|I\rangle_{1}|I\rangle_{2}
$$

The assumption of D17 would imply that the two measurements should not affect each other. In particular, the order of the two unitaries of (20) should be immaterial.

The joint distribution, conditioned on post-selecting $|\varphi\rangle$, is found to be

$$
\begin{aligned}
P_{\lambda_{1} \lambda_{2}} & \left(x_{1}, x_{2} \mid \varphi, \psi\right) \\
:= & P_{\lambda_{1} \lambda_{2}}(\varphi \mid \psi)^{-1}\left|\left\langle\varphi\left|\left\langle x_{1}\left|\left\langle x_{2}\left|e^{-i \lambda_{2} \hat{B} \hat{p}_{2}} e^{-i \lambda_{1} \hat{A} \hat{p}_{1}}\right| \psi\right\rangle\right| I\right\rangle_{1}\right| I\right\rangle_{2}\right|^{2} \\
= & G\left(x_{1}-\lambda_{1} \operatorname{Re}\left[\hat{A}_{w}\right]\right) G\left(x_{2}-\lambda_{2} \operatorname{Re}\left[\hat{B}_{w}\right]\right) \\
& \quad \times\left(1+\frac{\lambda_{1} \lambda_{2}}{2} \operatorname{Re}\left[(\hat{B} \hat{A})_{w}-\hat{A}_{w} \hat{B}_{w}\right] x_{1} x_{2}+O\left(\lambda_{1}^{2}\right)+O\left(\lambda_{2}^{2}\right)\right)
\end{aligned}
$$

7 I.e. that the disturbance of the intermediate measurements is insignificant when neglecting $O\left(\lambda_{1}^{2}\right)$ and $O\left(\lambda_{2}^{2}\right)$ terms. 
with

$$
(\hat{B} \hat{A})_{w}:=\frac{\langle\varphi|\hat{B} \hat{A}| \psi\rangle}{\langle\varphi \mid \psi\rangle} .
$$

In addition to the expected shifts corresponding to the weak values of $\hat{A}$ and $\hat{B}$ the last factor indicates that $x_{1}$ and $x_{2}$ have become correlated. This is not surprising, since the two measurements are performed on the same system. What is more interesting is that this factor does depend on the order of the measurements, contrary to the intuition from DI. Indeed, reversing the order corresponds to replacing $\operatorname{Re}\left[(\hat{B} \hat{A})_{w}\right] \rightarrow \operatorname{Re}\left[(\hat{A} \hat{B})_{w}\right]$ on the right hand side of (21), which in general makes a difference 8 This phenomena seems very difficult to understand under the assumption of DI9

As soon as one drops DI, it becomes clear that the order of the measurements should in general matter. To see this it is illuminating to work out the distribution for a joint measurement of $\hat{x}_{i}^{\prime}=2 \hat{p}_{i}$ :

$$
\begin{aligned}
P_{\lambda_{1} \lambda_{2}}\left(x_{1}^{\prime}, x_{2}^{\prime} \mid \varphi, \psi\right) & =G\left(x_{1}^{\prime}-\lambda_{1} \operatorname{Im}\left[\hat{A}_{w}\right]\right) G\left(x_{2}^{\prime}-\lambda_{2} \operatorname{Im}\left[\hat{B}_{w}\right]\right) \\
& \times\left(1+\frac{\lambda_{1} \lambda_{2}}{2} \operatorname{Re}\left[\hat{A}_{w} \hat{B}_{w}-(\hat{B} \hat{A})_{w}\right] x_{1}^{\prime} x_{2}^{\prime}+O\left(\lambda_{1}^{2}\right)+O\left(\lambda_{2}^{2}\right)\right)
\end{aligned}
$$

We notice that the result is very similar to (21). In particular this distribution will also depend on the order of the two interactions. In this case, however, it is no mystery how this dependence comes about. By the same reasoning as in Section 2 we can view (23) as describing a process where we first draw two random numbers $x_{1}^{\prime}, x_{2}^{\prime}$, then perturb the system according to

$$
\begin{aligned}
|\psi\rangle & \rightarrow e^{-i \lambda_{2} \hat{B} x_{2}^{\prime} / 2} e^{-i \lambda_{1} \hat{A} x_{1}^{\prime} / 2}|\psi\rangle \\
& =\left(1-i \lambda_{1} \frac{\hat{A}}{2} x_{1}^{\prime}-i \lambda_{2} \frac{\hat{B}}{2} x_{2}^{\prime}-\frac{\lambda_{1} \lambda_{2}}{2} \frac{\hat{B} \hat{A}}{2} x_{1}^{\prime} x_{2}^{\prime}+O\left(\lambda_{1}^{2}\right)+O\left(\lambda_{2}^{2}\right)\right)|\psi\rangle,
\end{aligned}
$$

and finally post-select on $|\varphi\rangle$. It is clear that the term of order $\lambda_{1} \lambda_{2}$ reflects the fact that we are perturbing with $\hat{A}$ first and then with $\hat{B}$, and that this is what is causing (23) to depend on the order of the unitaries.

\section{Conclusion}

Several phenomena connected with weak values appear paradoxical under the assumption of disturbance insignificance (DI). In particular we have discussed anomalous weak values and the fact that the order matters when performing

\footnotetext{
8 As long as $\hat{A}$ and $\hat{B}$ do not commute, $[\hat{A}, \hat{B}]$ is not proportional to the identity (we assume the system is finite dimensional). By the same argument as in Footnote 4 we can find states $|\varphi\rangle,|\psi\rangle$ such that $\operatorname{Re}\left[([\hat{A}, \hat{B}])_{w}\right] \neq 0$. But then $\operatorname{Re}\left[(\hat{B} \hat{A})_{w}\right] \neq \operatorname{Re}\left[(\hat{A} \hat{B})_{w}\right]$.

9 In Ref. 4 the fact that sequential weak measurements are sensitive to the ordering of the measurements is also discussed. But, since the authors have already decided according to other arguments that DI holds, they end up concluding that "[w]eak measurements are then still disturbing in some sense, although they do not disturb the state or later measurements".
} 
several weak measurements in sequence. As we have argued in Section 2 it is perfectly in accordance with standard quantum mechanics to drop DI as a general principle. Doing this allow us to understand these 'paradoxes' as mundane, albeit unintuitive, consequences of the ordinary laws of probability applied to experiments with weakly (but not negligibly) disturbing measurements and post-selection.

In this paper we have, for reasons of simplicity and concreteness, only considered weak measurement of the von Neumann type. The weak value can be obtained by much more general classes of measurement procedures. We refer the reader to the very brief sketch in Appendix D, Refs. 10,9 and references therein. There is no type of weak measurement which less disturbing (on average) than the von Neumann scheme[16, so our arguments and conclusions apply equally well to this broader class.

Acknowledgements Isabell Lubanski Ipsen is thanked for helpful comments on the manuscript, and Josh Combes is thanked for inspiring discussion. Lev Vaidman is also thanked for interesting correspondence.

\section{A State update and disturbance}

For an ideal classical measurement, it is usually assumed that the state of the system after interacting with the apparatus is the same as it was before the interaction. In contrast, the presence of measurement disturbance (or back-action) then means that this condition is not satisfied, i.e. that the meter somehow causes the system to change state.

The situation for quantum measurements is more subtle. Since it is the system that will have our interest, it is helpful to abstract away the 'inner workings' of the measurement procedure. As in the main text, let us concentrate on the von Neumann model. We thus consider an apparatus that takes a quantum system as input and produces a number $x$ and a quantum system as output. It can be completely characterized by two pieces of data. One is the probability $P(x \mid \psi)$ of result $x$ given the input $|\psi\rangle$. In our specific case $P(x \mid \psi)=P_{\lambda}(x \mid \psi)$ is given by Eq. (3). The second piece of data is the state of the output $\left|\chi_{\psi, x}\right\rangle$ conditioned on a specific result $x$,

$$
\left|\chi_{\psi, x}^{\lambda}\right\rangle:=P_{\lambda}(x \mid \psi)^{-1 / 2}\left\langle x\left|e^{-i \lambda \hat{A} \hat{p}}\right| \psi\right\rangle|I\rangle .
$$

In analogy with the classical case, we want to define quantum measurement disturbance as the change of the state of the system caused by the interaction with the meter (compare with e.g. Refs. 6] 18]). The question is how to compare the state before and after interaction.

One approach would be to look at how close $\left|\chi_{\psi, x}^{\lambda}\right\rangle$ is to $|\psi\rangle$. Expanding (25) for small $\lambda$ we find

$$
\left|\chi_{\psi, x}^{\lambda}\right\rangle=\left(1+\lambda\left[\hat{A}-\langle\hat{A}\rangle_{\psi}\right] \frac{x}{2}+O\left(\lambda^{2}\right)\right)|\psi\rangle .
$$

From this point of view the conclusion would thus be that the the back-action is of order $\lambda$ (unless $|\psi\rangle$ happens to be an eigenstate of $\hat{A}$ ), in conflict with DI.

Seemingly, there is just one way to avoid this conclusion, which is to understand a pure quantum states as only expressing partial knowledge about the system (similarly to how a probability distribution expresses partial knowledge about a classical random variable). Then it could be argued that the change $|\psi\rangle \rightarrow\left|\chi_{\psi, x}^{\lambda}\right\rangle$ is, at least partly, a form of Bayesian update (or conditioning). That is, the claim would be that some of the difference between $\left|\chi_{\psi, x}^{\lambda}\right\rangle$ and $|\psi\rangle$ reflects the fact that we learn something about the system by learning $x$, and should thus update our believes about the quantum system. From this point of view we can reformulate DI as

Disturbance Insignificance' (DI'): Any disturbance caused by the measurement process 
only shows up in the higher order (i.e. $O\left(\lambda^{2}\right)$ ) correction to the conditional state (26). Hence the first order correction must be understood as purely due to some form of Bayesian update. An interesting discussion of quantum Bayesian conditioning can be found in Section $\mathrm{V}$ of Ref. [18.

Let us mention that it is not possible to interpret the update $|\psi\rangle \rightarrow\left|\chi_{\psi, x}^{\lambda}\right\rangle$ purely as Bayesian conditioning. That is, the update necessarily introduces some amount of disturbance. Indeed, if $|\psi\rangle \rightarrow\left|\chi_{\psi, x}^{\lambda}\right\rangle$ were a kind of Bayesian update, then marginalizing over $x$ (i.e. 'unlearning' the measurement result) should give back the original state, but we actually find 10

$$
\rho_{\psi}^{\lambda}:=\int P_{\lambda}(x \mid \psi)\left|\chi_{\psi, x}^{\lambda}\right\rangle\left\langle\chi_{\psi, x}^{\lambda}\left|\mathrm{d} x=\int G\left(x^{\prime}\right) e^{-i \lambda \hat{A} x^{\prime} / 2}\right| \psi\right\rangle\langle\psi| e^{i \lambda \hat{A} x^{\prime} / 2} \mathrm{~d} x^{\prime}
$$

which is mixed (unless $|\psi\rangle$ is an eigenstate of $\hat{A}$ ), and thus not equal to the initial state $|\psi\rangle\langle\psi|$. It is a general result that any quantum measurement must be disturbing in this sense $6 \begin{array}{lll}6 & 18\end{array}$

The fact that the difference between $\rho_{\psi}^{\lambda}$ and $|\psi\rangle\langle\psi|$ is solely due to back-action is used in Section 2 to quantify the amount of disturbance. Indeed, the difference between the overall post-selection probability $P_{\lambda}(\varphi \mid \psi)$ (see Eq. (10) ) and its unperturbed value $|\langle\varphi \mid \psi\rangle|^{2}$ can be written as

$$
P_{\lambda}(\varphi \mid \psi)-|\langle\varphi \mid \psi\rangle|^{2}=\left\langle\rho_{\psi}^{\lambda}-\mid \psi\right\rangle\langle\psi \mid\rangle_{\varphi}
$$

\section{B Disturbance Insignificance in the literature}

We claim in the introduction that a number of works in the literature apparently conclude or postulate that the disturbance of weak measurements can be neglected. The subtle nature of the subject matter means that there is a substantial risk of misunderstandings. With that in mind we will quote some relevant passages from these papers. After the quotes we make a couple of brief remarks.

- (Vaidman, 1996, p. 899) 23]: "I propose to consider the standard measuring procedure $[\ldots]$ in which we weaken the interaction in such a way that the state of the quantum system is not changed significantly during the interaction."

- (Resch, Lundeen \& Steinberg, 2004, p. 125) [20]: "In particular, [the weak measurement strategy] makes it possible to contemplate the behavior of a system defined both by state preparation and by a later post-selection, without significant disturbance of the system in the intervening period."

- (Tollaksen, 2007, abstract) 22]: "When measurements are performed which do not disturb the pre- and post-selection (i.e. weak measurements) [...]"

- (Tollaksen, 2007, p. 9063) 22]: "[...] we have now shown that when considered as a limiting process, the disturbance goes to zero more quickly than the shift in the measuring device, which means for a large enough ensemble, information (e.g. the expectation value) can be obtained even though not even a single particle is disturbed."

- (Hofmann, 2010, abstract) [14]: "[...] weak measurements have negligible back action $[\ldots] "$

- (Hofmann, 2010, p. 2) 14]: "For very small measurement strengths $\epsilon$, the effects of the quantum state on the measurement probabilities is linear in $\epsilon$ while the measurement back action is quadratic in $\epsilon$. It is therefore possible to realize quantum state tomography with negligible back action."

- (Dressel \& Jordan, 2012, p. 7)[11]: "[the real part of the weak value] can be interpreted as an idealized limit point for the average of $\hat{A}$ in the initial state $\hat{\rho}_{i}$ that has been conditioned on the postselection $\hat{P}_{f}$ without any appreciable intermediate measurement disturbance."

10 The map $|\psi\rangle\left\langle\psi\left|\mapsto \int P_{\lambda}(x \mid \psi)\right| \chi_{\psi, x}^{\lambda}\right\rangle\left\langle\chi_{\psi, x}^{\lambda}\right| \mathrm{d} x$ is sometimes called a non-selective update.

11 A precise statement is: If $\rho_{\psi}=|\psi\rangle\langle\psi|$ for all $\psi$, then the probability $P(x \mid \psi)$ is independent of $\psi$. 
- (Bednorz, Franke \& Belzig, 2013, abstract) 4]: "We show that it is possible to define general weak measurements, which are noninvasive: the disturbance becomes negligible as the measurement strength goes to zero."

- (Bednorz, Franke \& Belzig, 2013, p. 9) 4]: "[...] weak measurements (both classical and quantum) are noninvasive in a stronger sense: their disturbance vanishes as $g^{2}$ regardless of the type of measurements before/after.'

- (Vaidman et al., 2017, p. 2) 24]: "The change in the other systems [e.g. a meter] should be large enough to be seen, but the back action on the system should be small enough, such that the change in the two-state vector describing the system can be neglected. Since we allow an unlimited ensemble of experiments with identical pre- and postselection, the required limits are achievable."

- (Cohen, 2017, p. 1263)[7: "[...] for $\epsilon \ll 1$, which is indeed justified in the weak measurement regime, then the fidelity $\sqrt{12}$ is 1 up to $O\left(\epsilon^{2}\right)$. Therefore, by definition, the state has been negligibly disturbed, and this is now a precise claim."

- (Cohen, 2017, p. 1264) 7]: "[...] weak measurements are non-invasive, that is, the probability of evolving the initial state to an orthogonal state through a weak measurement decreases like $g^{2}$. This property significantly limits the amount of backaction [...]"

Refs. 22 144,7 all conclude that the disturbance is of second order in the interaction strength using variants of the argument discussed, and found unconvincing, in Section 2 The arguments of Ref. 11 are not discussed in this paper, but in Appendix D we address some related ideas by the same authors. It is not clear (to this author) what definition is being referred to in the first quote from Ref. [7.

\section{Collective weak measurements}

In the usual protocol for measuring the weak value the variance of $x$ is large compared to the shift $\lambda \operatorname{Re} \hat{A}_{w}$. We thus have to repeat the experiment many times to get a good estimate. Another possibility is to let a single meter interact with a large number $N$ of identically prepared systems. [3] In this case both the uncertainty of the (single) measurement result and the back-action on each system can, in principle, be made as small as one would like. It could thus seem as if this would exclude the possibility of the kind of statistical effects we have discussed so far. An analysis along the lines of Section 2 however, shows that the presence of significant disturbance, if anything, is more evident.

We again consider the interaction to be of von Neumann type,

$$
|\psi\rangle^{N}|I\rangle \rightarrow e^{-i \lambda \bar{A} \hat{p}}|\psi\rangle^{N}|I\rangle,
$$

but now $\bar{A}$ is the averaged observable

$$
\bar{A}:=N^{-1} \sum_{n=1}^{N} \hat{A}_{n}
$$

and we abbreviate

$$
|\psi\rangle^{N}:=|\psi\rangle_{1}|\psi\rangle_{2} \cdots|\psi\rangle_{N} .
$$

The conditional distribution of $x$ is

$$
P_{\lambda}\left(x \mid \varphi^{N}, \psi^{N}\right)=P_{\lambda}\left(\varphi^{N} \mid \psi^{N}\right)^{-1}\left|\left\langle x\left|\left(\left\langle\varphi\left|e^{-i \lambda N^{-1} \hat{A} \hat{p}}\right| \psi\right\rangle\right)^{N}\right| I\right\rangle\right|^{2} .
$$

12 The fidelity between the initial pure state $\psi$ and the state of the system after interaction
with the meter is equal to the post-selection probability $P_{\lambda}(\psi \mid \psi)$. In the case of a von
Neumann measurements we find $1-\lambda^{2}\left(\left\langle\hat{A}^{2}\right\rangle_{\psi}-\left[\langle\hat{A}\rangle_{\psi}\right]^{2}\right) / 4$ by substituting $\varphi \rightarrow \psi$ in (10). 
If we now keep $\lambda$ fixed (not necessarily small), but send $N \rightarrow \infty$, we can expand 13

$$
\begin{aligned}
\left(\left\langle\varphi\left|e^{-i \lambda N^{-1} \hat{A} \hat{p}}\right| \psi\right\rangle\right)^{N} & =(\langle\varphi \mid \psi\rangle)^{N}\left[1-i \lambda N^{-1} \hat{A}_{w} \hat{p}+O\left(N^{-2}\right)\right]^{N} \\
& =(\langle\varphi \mid \psi\rangle)^{N}\left[e^{-i \lambda \hat{A}_{w} \hat{p}}+O\left(N^{-1}\right)\right]
\end{aligned}
$$

leading to

$$
\begin{aligned}
P\left(x \mid \varphi^{N}, \psi^{N}\right) & =P_{\lambda}\left(\varphi^{N} \mid \psi^{N}\right)^{-1}|\langle\varphi \mid \psi\rangle|^{2 N}(2 \pi)^{-1 / 2}\left|e^{-\left(x-\lambda \hat{A}_{w}\right)^{2} / 4}+O\left(N^{-1}\right)\right|^{2} \\
& =G\left(x-\lambda \operatorname{Re} \hat{A}_{w}\right)+O\left(N^{-1}\right)
\end{aligned}
$$

We again find a shift proportional to the weak value, but now without any condition that the constant of proportionality $\lambda$ is small.

Turning to the question of back-action, we first note that the strength of the coupling to each individual system is $N^{-1} \lambda$, which is certainly small in the limit we are considering. However, a more relevant quantity in connection with the conditional distribution of $x$ is the overall post-selection probability

$$
P_{\lambda}\left(\varphi^{N} \mid \psi^{N}\right)=|\langle\varphi \mid \psi\rangle|^{2 N}\left[e^{\lambda^{2}\left(\operatorname{Im} \hat{A}_{w}\right)^{2} / 2}+O\left(N^{-1}\right)\right]
$$

which is not in general close to the unperturbed value of $|\langle\varphi \mid \psi\rangle|^{2 N}$. We see that, when looking at all $N$ systems as a whole, the disturbance from the interaction 29] is simply large! It thus seems difficult to argue that it nevertheless should be considered insignificant.

As for the ordinary weak measurement, it is instructive to work out the conditional distribution for a measurement of $\hat{x}^{\prime}=2 \hat{p}$. We find

$$
\begin{aligned}
P\left(x^{\prime} \mid \varphi^{N}, \psi^{N}\right) & =P_{\lambda}\left(\varphi^{N} \mid \psi^{N}\right)^{-1}\left|\left\langle x^{\prime} \mid I\right\rangle\right|^{2}\left|\left\langle\varphi\left|e^{-i \lambda N^{-1} \hat{A} x^{\prime} / 2}\right| \psi\right\rangle\right|^{2 N} \\
& =P_{\lambda}\left(\varphi^{N} \mid \psi^{N}\right)^{-1} G\left(x^{\prime}\right)|\langle\varphi \mid \psi\rangle|^{2 N}\left[e^{\lambda \operatorname{Im}\left[\hat{A}_{w}\right] x^{\prime}}+O\left(N^{-1}\right)\right] \\
& =G\left(x^{\prime}-\lambda \operatorname{Im} A_{w}\right)+O\left(N^{-1}\right)
\end{aligned}
$$

This shift is entirely due to the back-action, but still of the same order of magnitude as that of $x$ (Eq. (34))!

We conclude that, also in the case of collective weak measurements, there is no indication that the disturbance of the intermediate measurement is negligible.

\section{Disturbance and the Lindblad super-operator}

Here we make some remarks on the claims about disturbance in Refs. 12,8] (some related ideas can be found in Ref. 11]). We have to begin by making some technical definitions. First we write the joint probability as

$$
P_{\lambda}(x, \varphi \mid \psi)=\left|\left\langle\varphi\left|\hat{M}_{x}^{\lambda}\right| \psi\right\rangle\right|^{2},
$$

where $\hat{M}_{x}^{\lambda}$ is an operator acting on the system Hilbert space. In the von Neumann case we get the equation

$$
\left|\left\langle\varphi\left|\left\langle x\left|e^{-i \lambda \hat{A} \hat{p}}\right| \psi\right\rangle\right| I\right\rangle\right|^{2}=\left|\left\langle\varphi\left|\hat{M}_{x}^{\lambda}\right| \psi\right\rangle\right|^{2},
$$

13 We are being somewhat lax in dealing with the unbounded operator $\hat{p}$, a more careful treatment can be found in e.g. Ref. [3]. 
so we can take $\hat{M}_{x}^{\lambda}$ to be given by (Eq. (38) does not uniquely fix $\hat{M}$, since a phase $\hat{M}_{x} \rightarrow$ $e^{i f_{x}} \hat{M}_{x}$ drops out)

$$
\begin{aligned}
\hat{M}_{x}^{\lambda} & =\left\langle x\left|e^{-i \lambda \hat{A} \hat{p}}\right| I\right\rangle \\
& =\left\langle x\left|\left[1-i \lambda \hat{A} \hat{p}-\frac{1}{2} \lambda^{2} \hat{A}^{2} \hat{p}^{2}+O\left(\lambda^{3}\right)\right]\right| I\right\rangle \\
& =\sqrt{G(x)}\left[1+\lambda \frac{x}{2} \hat{A}+\frac{\lambda^{2}}{2}\left(\frac{x^{2}}{4}-\frac{1}{2}\right) \hat{A}^{2}+O\left(\lambda^{3}\right)\right] .
\end{aligned}
$$

We will not assume that $\hat{M}_{x}^{\lambda}$ is Hermitian, although it happens to be in this specific example.

The key step of Refs. 12,8] is to rewrite (37) as

$$
P_{\lambda}(x, \varphi \mid \psi)=P_{\lambda}^{w}(x, \varphi \mid \psi)+\mathcal{E}_{\lambda}(x, \varphi \mid \psi),
$$

with $P^{w}$ define by $(\{\cdot, \cdot\}$ denotes the anti-commutator $)$

$$
P_{\lambda}^{w}(x, \varphi \mid \psi):=|\langle\varphi \mid \psi\rangle|^{2} \operatorname{Re}\left[\left(\left[\hat{M}_{x}^{\lambda}\right]^{\dagger} \hat{M}_{x}^{\lambda}\right)_{w}\right]=\frac{1}{2}\left\langle\left\{\left[\hat{M}_{x}^{\lambda}\right]^{\dagger} \hat{M}_{x}^{\lambda},|\varphi\rangle\langle\varphi|\right\}\right\rangle_{\psi} .
$$

It follows that the 'error term' is given by

$$
\mathcal{E}_{\lambda}(x, \varphi \mid \psi)=\left\langle\mathcal{L}\left[\hat{M}_{x}^{\lambda}\right](|\varphi\rangle\langle\varphi|)\right\rangle_{\psi},
$$

with the Lindblad super-operator 14 defined as

$$
\mathcal{L}[\hat{M}](\hat{O})=\frac{1}{2}\left(\left[\hat{M}^{\dagger}, \hat{O}\right] \hat{M}+\hat{M}^{\dagger}[\hat{O}, \hat{M}]\right)
$$

It is now observed that, if $\mathcal{E}$ can be neglected in the $\lambda \rightarrow 0$ limit, then $P_{\lambda}(x, \varphi \mid \psi) \approx$ $P_{\lambda}^{w}(x, \varphi \mid \psi)$ and so the conditional expectation value of $x$ is approximately 12$]$

$$
\frac{\int x P_{\lambda}^{w}(x, \varphi \mid \psi) \mathrm{d} x}{\int P_{\lambda}^{w}(x, \varphi \mid \psi) \mathrm{d} x}=\frac{|\langle\varphi \mid \psi\rangle|^{2} \int x \operatorname{Re}\left[\left(\left[\hat{M}_{x}^{\lambda}\right]^{\dagger} \hat{M}_{x}^{\lambda}\right)_{w}\right] \mathrm{d} x}{|\langle\varphi \mid \psi\rangle|^{2}}=\lambda \operatorname{Re} \hat{A}_{w}+O\left(\lambda^{2}\right) .
$$

Here we have used (in the von Neumann case there are actually no higher order corrections)

$$
\int x\left[\hat{M}_{x}^{\lambda}\right]^{\dagger} \hat{M}_{x}^{\lambda} \mathrm{d} x=\lambda \hat{A}+O\left(\lambda^{2}\right)
$$

and

$$
\int\left[\hat{M}_{x}^{\lambda}\right]^{\dagger} \hat{M}_{x}^{\lambda} \mathrm{d} x=\hat{1}
$$

to simplify the numerator and denominator. This argument shows that any weak measure ment (i.e. not necessarily of von Neumann type) that can be parametrized by operators $\hat{M}_{x}^{\lambda}$ satisfying (45) and (46) will result in $\lambda \operatorname{Re} \hat{A}_{w}$ as the conditional expectation value, as long as the error terms can be neglected 15

\footnotetext{
14 As is mentioned in both references, Lindblad terms are association with dissipative dynamics of open quantum systems. It is, however, not clear what the significance of the fact that $\mathcal{E}$ can be written using the Lindblad super-operator is.

15 We have simplified the presentation somewhat compared to Refs. 12 8]. There Eq. 455 is, up to changes in notation, written as $\int \alpha_{x}\left[\hat{M}_{x}^{\lambda}\right]^{\dagger} \hat{M}_{x}^{\lambda} \mathrm{d} x=\hat{A}$, where the 'contextual values' $\alpha_{x}$ can have complicated dependence on $x$ and $\lambda$. The additional generality is not important for the argument we are making.
} 
The connection with back-action come from the claim 12 8 16 which we will call Generalized Disturbance Insignificance (GDI): If the error term $\mathcal{E}_{\lambda}(x, \varphi \mid \psi)$ can be neglected 17 then the disturbance caused by the intermediate measurement is insignificant.

As the names suggests, DI is a special case of GDI. To see this, we just need to check that the error term can be neglected for weak von Neumann measurements. Inserting (39) into (42) we find 18

$$
\mathcal{E}_{\lambda}(x, \varphi \mid \psi)=|\langle\varphi \mid \psi\rangle|^{2} G(x)\left[\frac{\lambda^{2}}{4}\left(\left|\hat{A}_{w}\right|^{2}-\operatorname{Re}\left[\left(\hat{A}^{2}\right)_{w}\right]\right) x^{2}+O\left(\lambda^{3}\right)\right]
$$

which is indeed $O\left(\lambda^{2}\right)$. This in turn means that the paradoxical consequences of adopting DI also follow from adopting GDI. In Ref. [12] it is remarked that

$$
P_{\lambda}(\varphi \mid \psi)=\int P_{\lambda}(x, \varphi \mid \psi) \mathrm{d} x \approx \int P_{\lambda}^{w}(x, \varphi \mid \psi) \mathrm{d} x=|\langle\varphi \mid \psi\rangle|^{2}
$$

whenever $\mathcal{E}$ can be neglected. This is taken as evidence that "[t]he Lindblad operation indicates disturbance that the intermediate measurement introduces to the measurement sequence." 12 But, as discussed in Section 2 the approximate equality $P_{\lambda}(\varphi \mid \psi) \approx|\langle\varphi \mid \psi\rangle|^{2}$ does not imply that the disturbance is insignificant.

To summarize, GDI is, like DI, an additional postulate which does not follow from ordinary quantum mechanics. We do not know of any convincing arguments in favor of GDI, while the arguments against DI works equally well against GDI.

\section{References}

1. Aharonov, Y., Albert, D.Z., Vaidman, L.: How the result of a measurement of a component of the spin of a spin- $1 / 2$ particle can turn out to be 100 . Phys. Rev. Lett. 60, 1351-1354 (1988). DOI 10.1103/PhysRevLett.60.1351. URL http://link.aps.org/doi/10.1103/PhysRevLett.60.1351

2. Aharonov, Y., Botero, A.: Quantum averages of weak values. Phys. Rev. A 72, 052111 (2005). DOI 10.1103/PhysRevA.72.052111. URL http://link.aps.org/doi/10.1103/PhysRevA.72.052111

3. Aharonov, Y., Vaidman, L.: Properties of a quantum system during the time interval between two measurements. Phys. Rev. A 41, 11-20 (1990). DOI 10.1103/PhysRevA. 41.11. URL http://link.aps.org/doi/10.1103/PhysRevA.41.11

4. Bednorz, A., Franke, K., Belzig, W.: Noninvasiveness and time symmetry of weak measurements. New Journal of Physics 15(2), 023043 (2013). DOI 10.1088/1367-2630/15/ 2/023043. URL https://doi.org/10.1088/1367-2630/15/2/023043

5. Bub, J., Brown, H.: Curious properties of quantum ensembles which have been both preselected and post-selected. Phys. Rev. Lett. 56, 2337-2340 (1986). DOI 10.1103/PhysRevLett.56.2337. URL https://link.aps.org/doi/10.1103/PhysRevLett.56.2337

6. Busch, P.: "No information without disturbance": Quantum limitations of measurement. In: Quantum Reality, Relativistic Causality, and Closing the Epistemic Circle, The Western Ontario Series in Philosophy of Science, vol. 73, pp. 229-256. Springer Netherlands (2009). DOI 10.1007/978-1-4020-9107-0\_13. URL http://dx.doi.org/10.1007/978-1-4020-9107-0_13

16 It is expressed most clearly in Ref. [8] as "[w]hen the error terms $\mathcal{E}$ are small enough to be neglected [...] (meaning that the initial system state is negligibly perturbed), the real part of the weak value [...] is unambiguously recovered as the measured conditioned estimate for $\hat{A}$, verifying our derivation of this real part as a best estimate."

17 Since we assume that we can expand in integer powers of $\lambda$, that $\mathcal{E}_{\lambda}(x, \varphi \mid \psi)$ can be neglected means that it is $O\left(\lambda^{2}\right)$.

18 The similarity with 10 is no coincidence, since we have the general relation $\int \mathrm{d} x \mathcal{E}_{\lambda}(x, \varphi \mid \psi)=P_{\lambda}(\varphi \mid \psi)-|\langle\varphi \mid \psi\rangle|^{2}$. 
7. Cohen, E.: What Weak Measurements and Weak Values Really Mean: Reply to Kastner. Foundations of Physics 47(10), 1261-1266 (2017). DOI 10.1007/s10701-017-0107-2. URL https://doi.org/10.1007/s10701-017-0107-2

8. Dressel, J.: Weak values as interference phenomena. Phys. Rev. A 91, 032116 (2015). DOI 10.1103/PhysRevA.91.032116. URL http://link.aps.org/doi/10.1103/PhysRevA.91.032116

9. Dressel, J., Jordan, A.: Contextual-value approach to the generalized measurement of observables. Phys. Rev. A 85, 022123 (2012). DOI 10.1103/PhysRevA.85.022123. URL http://link.aps.org/doi/10.1103/PhysRevA.85.022123

10. Dressel, J., Jordan, A.: Sufficient conditions for uniqueness of the weak value. Journal of Physics A: Mathematical and Theoretical 45(1), 015304 (2012). URL http://stacks.iop.org/1751-8121/45/i=1/a=015304

11. Dressel, J., Jordan, A.N.: Significance of the imaginary part of the weak value. Phys. Rev. A 85, 012107 (2012). DOI 10.1103/PhysRevA.85.012107. URL http://link.aps.org/doi/10.1103/PhysRevA.85.012107

12. Dressel, J., Jordan, A.N.: Quantum instruments as a foundation for both states and observables. Phys. Rev. A 88, 022107 (2013). DOI 10.1103/PhysRevA.88.022107. URL http://link.aps.org/doi/10.1103/PhysRevA.88.022107

13. Ferrie, C., Combes, J.: How the result of a single coin toss can turn out to be 100 heads. Phys. Rev. Lett. 113, 120404 (2014). DOI 10.1103/PhysRevLett.113.120404. URL http://link . aps .org/doi/10.1103/PhysRevLett.113.120404

14. Hofmann, H.F.: Complete characterization of post-selected quantum statistics using weak measurement tomography. Phys. Rev. A 81, 012103 (2010). DOI 10.1103/ PhysRevA.81.012103. URL http://link.aps.org/doi/10.1103/PhysRevA.81.012103

15. Hu, W.: The curious quantum mechanics of pre-and post-selected ensembles. Foundations of physics 20(4), 447-458 (1990). DOI 10.1007/BF00731712. URL https://doi.org/10.1007/BF00731712

16. Ipsen, A.C.: Disturbance in weak measurements and the difference between quantum and classical weak values. Phys. Rev. A 91, 062120 (2015). DOI 10.1103/PhysRevA. 91.062120. URL https://link.aps.org/doi/10.1103/PhysRevA.91.062120

17. Kastner, R.E.: Demystifying Weak Measurements. Foundations of Physics 47(5), 697-707 (2017). DOI 10.1007/s10701-017-0085-4. URL https://doi.org/10.1007/s10701-017-0085-4

18. Leifer, M.S., Spekkens, R.W.: Towards a formulation of quantum theory as a causally neutral theory of bayesian inference. Phys. Rev. A 88, 052130 (2013). DOI 10.1103/ PhysRevA.88.052130. URL https://link.aps.org/doi/10.1103/PhysRevA .88.052130

19. Mitchison, G., Jozsa, R., Popescu, S.: Sequential weak measurement. Phys. Rev. A 76, 062105 (2007). DOI 10.1103/PhysRevA.76.062105. URL http://link.aps.org/doi/10.1103/PhysRevA.76.062105

20. Resch, K., Lundeen, J., Steinberg, A.: Experimental realization of the quantum box problem. Physics Letters A 324(2-3), 125-131 (2004). DOI http://dx.doi.org/10.1016/j.physleta.2004.02.042. URL http://www.sciencedirect.com/science/article/pii/S0375960104002506

21. Resch, K., Steinberg, A.: Extracting joint weak values with local, single-particle measurements. Physical review letters 92(13), 130402 (2004). DOI 10.1103/PhysRevLett. 92.130402. URL https://doi.org/10.1103/PhysRevLett.92.130402

22. Tollaksen, J.: Pre- and post-selection, weak values and contextuality. Journal of Physics A: Mathematical and Theoretical 40(30), 9033 (2007). URL https://doi.org/10.1088/1751-8113/40/30/025

23. Vaidman, L.: Weak-measurement elements of reality. Foundations of Physics 26(7), 895906 (1996). DOI 10.1007/BF02148832. URL http://dx.doi.org/10.1007/BF02148832

24. Vaidman, L., Ben-Israel, A., Dziewior, J., Knips, L., Weißl, M., Meinecke, J., Schwemmer, C., Ber, R., Weinfurter, H.: Weak value beyond conditional expectation value of the pointer readings. Phys. Rev. A 96, 032114 (2017). DOI 10.1103/PhysRevA.96.032114. URL https://link.aps.org/doi/10.1103/PhysRevA.96.032114 